\title{
GMR
}

\section{Detection and characterization of carbendazim resistance in Sclerotinia sclerotiorum isolates from oilseed rape in Anhui Province of China}

\author{
D. Xu*, Y. Pan*, H. Zhang, X. Li, Y. Dai, S. Cao and Z. Gao \\ Department of Plant Pathology, School of Plant Protection, \\ Anhui Agricultural University, Hefei, China
}

${ }^{*}$ These authors contributed equally to this study.

Corresponding author: Z. Gao

E-mail: gaozhimou@126.com

Genet. Mol. Res. 14 (4): 16627-16638 (2015)

Received August 23, 2015

Accepted October 11, 2015

Published December 11, 2015

DOI http://dx.doi.org/10.4238/2015.December.11.10

\begin{abstract}
Stem rot caused by Sclerotinia sclerotiorum is a devastating disease of oilseed rape (Brassica napus) in Anhui Province of China. The fungicide carbendazim (methyl benzimidazole-2-yl carbamate; MBC) has been used to control this fungal disease since the 1980s. In the present study, 74 isolates of $S$. sclerotiorum from 13 regions of Anhui were collected, and the sensitivities of these isolates to MBC were examined to monitor fungicide resistance. We found that 22 of the 74 isolates showed resistance to $\mathrm{MBC}$, indicating that $S$. sclerotiorum has developed resistance in parts of Anhui Province. PCR assays and DNA sequence analysis showed that isolates with high MBC resistance had a point mutation at position 198 in the $\beta$-tubulin gene that caused a glutamic acid-to-alanine change in the protein. The $\beta$-tubulin gene in low-resistance isolates did not have the mutation. These results indicate that the mutation in $\beta$-tubulin gene may be associated with high MBC resistance in $S$. sclerotiorum. The present study also found no correlation between MBC resistance and pathogenicity of $S$. sclerotiorum isolates, suggesting that the pathogenicity of $S$. sclerotiorum
\end{abstract}


isolates on oilseed rape did not vary with $\mathrm{MBC}$ resistance status.

Key words: Sclerotinia sclerotiorum; Carbendazim resistance; Molecular mechanism; Pathogenicity; Oilseed rape

\section{INTRODUCTION}

Sclerotinia sclerotiorum (Lib.) de Bary is a cosmopolitan fungal pathogen that attacks more than 400 species of plants, including many important crops such as soybean, bean, sunflower, canola, and oilseed rape (Purdy, 1979; Boland and Hall, 1994; Zhang et al., 2014). Sclerotinia stem rot in rape as a consequence of $S$. sclerotiorum attack results in serious loss of yield and quality in oilseed rape (Boland and Hall, 1994; Xu et al., 2014). In recent decades, the disease has caused tremendous losses to rapeseed production in Canada (Bardin and Huang, 2001), U.S.A. (Purdy, 1979; Bolton et al., 2006), Australia (Letham et al., 1976) and China (Gao et al., 2014). Breeding programs for disease resistance are hampered by limited gene resources (Lu, 2003; Zhao and Meng, 2003). As a consequence, Sclerotinia stem rot continues to impose serious limitations on oilseed rape production all over the world. In practice, the application of fungicides is the principal response in most oilseed rape growing regions for managing Sclerotinia stem rot. The benzimidazole fungicide, carbendazim (methyl benzimidazole-2-yl carbamate; $\mathrm{MBC}$ ), has been widely used to control this disease in China since the 1980s. However, the effectiveness of control achieved by the fungicide has declined continuously in some regions due to the development of MBC resistance (Shi et al., 2000; Zhang et al., 2003). Recently, MBC resistance in S. sclerotiorum has led to disease control failures; MBC resistance is widespread throughout Jiangsu Province with an estimated resistance frequency of $29.54 \%$ (Ma et al., 2009). It appears that the repeated and intensive applications of MBC have caused the emergence of resistant strains of S. sclerotiorum in fields in Jiangsu (Wang et al., 2014). However, the sensitivity of S. sclerotiorum isolates to MBC in Anhui Province is currently unknown. Thus, it is essential to investigate MBC sensitivity in S. sclerotiorum isolates from oilseed rape in Anhui Province in order to assess the risk of fungicide resistance.

In the present study, MBC resistance in $S$. sclerotiorum isolates from oilseed rape in the main growing areas of Anhui Province was measured and the resistance mechanisms were explored in an attempt to provide experimental evidence for the management of MBC resistance in S. sclerotiorum and the integrated control of Sclerotinia stem rot in rapeseed.

\section{MATERIAL AND METHODS}

\section{Fungicides and media}

Technical grade (98\%) carbendazim (methyl benzimidazole-2-yl carbamate; MBC) was provided by Anhui Academy of Chemistry and Industry (Hefei, China) and was dissolved in $0.1 \mathrm{M}$ hydrochloric acid $(\mathrm{HCl})$ at $10 \mathrm{mg} / \mathrm{mL}$ as a stock solution.

Potato dextrose agar medium (PDA) was made from $200 \mathrm{~g}$ potato, $20 \mathrm{~g}$ agar and $20 \mathrm{~g}$ dextrose per liter of distilled water. Potato dextrose broth medium (PDB) was made from $200 \mathrm{~g}$ potato and $20 \mathrm{~g}$ dextrose per liter distilled water (Perez et al., 1992). These two media were used for routine culture and in the experiments to determine the sensitivity of $S$. sclerotiorum isolates to the fungicide.

\section{Origin and collection of isolates}

The isolates of S. sclerotiorum were collected from oilseed rape fields in Hefei, Tongcheng, 
Jingde, Ningguo, Lujiang, Wuwei, Wuhu, Shouxian, Lu'an, Nanling, Xuanzhou, Fanchang, and Wangjiang of Anhui Province in China. The oilseed rape fields used for collection of the isolates were separated from each other by more than $10 \mathrm{~km}$. In each field, several plants with symptoms of Sclerotinia stem rot were randomly collected, air-dried, placed in paper envelopes, and stored at $-4^{\circ} \mathrm{C}$. All isolates were derived from individual sclerotia collected from the oilseed rape plants. The sclerotia were surface sterilized in $0.1 \%$ sodium hypochlorite for $5 \mathrm{~min}$, rinsed in sterile distilled water for $30 \mathrm{~s}$, bisected and one of the two halves was placed on a PDA plate. PDA plates were incubated for 3 days at $25^{\circ} \mathrm{C}$ in a growth chamber (12 h photoperiod). Pure cultures were obtained by transfer of a single sclerotium and maintained on PDA slants at $4^{\circ} \mathrm{C}$ for 2 to 4 weeks. In all, 74 isolates were collected throughout Anhui Province.

\section{Determination of sensitivity of $S$. sclerotiorum to MBC}

The sensitivity of the $S$. sclerotiorum isolates to $\mathrm{MBC}$ was assayed using the colony growth rate method (Ma et al., 2009). A $5 \mathrm{~mm}$ mycelial plug was taken from the edge of a 3-day-old colony and placed onto the center of a PDA plate treated with $0.005,0.01,0.05,0.1,0.5,1,5,10,20,40$, $80,160,320$, or $640 \mathrm{mg} / \mathrm{L} \mathrm{MBC}$. For each isolate, three replicate plates per concentration were used. In the control group, distilled water at the same volume was added into the melted PDA and the mycelial plugs were cultured on the PDA plates. All the dishes were cultured for 2 days at $25^{\circ} \mathrm{C}$ in the dark. The cross method was employed to measure colony diameters at intervals of $24 \mathrm{~h}$. The following formula was used to calculate the growth inhibition rate:

$$
(\%)=\frac{(\text { control colony diameter }- \text { processed colony diameter })}{(\text { control colony diameter }-5)} \times 100
$$

The growth inhibition rate was then transformed into a probability value of inhibition using the Response Rate \& Probability Value conversion table and the MBC concentration was transformed into a logarithm (X) (Wang et al. 2014). The formula $Y=a+b$ X was used to calculate the toxicity of MBC to $S$. sclerotiorum through the regression method. The $\mathrm{EC}_{50}$ value calculated from this formula was used to evaluate the degree of resistance of all colonies to MBC. The sensitivity of $S$. sclerotiorum isolates to $\mathrm{MBC}$ was classified into five types as suggested by Yang et al. (2004): sensitive $\left(\mathrm{MBC}^{\mathrm{S}}\right)$, with $\mathrm{EC}_{50}<0.5 \mathrm{mg} / \mathrm{L}$; low resistance $\left(\mathrm{MBC}^{\mathrm{LR}}\right)$, with an $\mathrm{EC}_{50}$ of $0.5-5$ $\mathrm{mg} / \mathrm{L}$; medium resistance $\left(\mathrm{MBC}^{\mathrm{MR}}\right)$, with an $\mathrm{EC}_{50}$ of $5-50 \mathrm{mg} / \mathrm{L}$; high resistance $\left(\mathrm{MBC}^{\mathrm{HR}}\right)$, with an $\mathrm{EC}_{50}$ of $50-500 \mathrm{mg} / \mathrm{L}$; and super-high resistance $\left(\mathrm{MBC}^{\mathrm{VHR}}\right)$, with $\mathrm{EC}_{50}>500 \mathrm{mg} / \mathrm{L}$.

The experimental data were analyzed by analyses of variance (ANOVA) using SAS GLM (SAS Institute, Inc., Cary, NC, USA). When the ANOVA was significant $(P<0.05)$, means were separated using Fisher's Protected Least Significant Difference (PLSD). Differences between the $M B C$ resistant and $M B C$ sensitive groups of isolates were analyzed using $t$-tests.

\section{Mycelial culture}

Eleven $S$. sclerotiorum isolates with different resistance levels were selected and cultured on PDA for 3 days: sensitive isolates FC, SX-11-1, and SX-A; low resistance isolates XZ, NGA15, SX-3, JDA13, and FD; and high resistance isolates SX4, SX2, and NGA5. Mycelia from each colony were transferred to $60 \mathrm{~mL} P D B$ and cultured for 6 days at $25^{\circ} \mathrm{C}$. The mycelia were then collected with sterilized gauze and filters and washed twice in sterile water. Next, the mycelia were dried using a freezing vacuum dryer and stored at $-20^{\circ} \mathrm{C}$ for later use. 


\section{Extraction of genomic DNA}

Genomic DNA was extracted using the cetyltrimethylammonium bromide method (Stewart and Via, 1993), separated on a $1 \%$ agarose gel, and stored at $-20^{\circ} \mathrm{C}$ for later use.

\section{PCR amplification of $\beta$-tubulin gene}

The following are sequences of the forward and reverse primers designed by Shanghai Invitrogen Biotechnology Co., Ltd. according to exon sequences of the S. sclerotiorum $\beta$-tubulin gene: forward primer ATGCGTGAGATCGTATGTATATCT and reverse primer: ACTAATGCAAGATCAGT AACCAGT. $\beta$-tubulin was isolated respectively from the genomic DNA of the 11 isolates with resistance to carbendazim using the forward primer and the reverse primer under following conditions: $94^{\circ} \mathrm{C} 5 \mathrm{~min} ; 94^{\circ} \mathrm{C} 30 \mathrm{~s}, 54^{\circ} \mathrm{C} 30 \mathrm{~s}, 72^{\circ} \mathrm{C} 2 \mathrm{~min}, 35$ cycles; $72^{\circ} \mathrm{C} 7 \mathrm{~min}$; preserved at $4^{\circ} \mathrm{C}$. The amplified products were sequenced after T-vector connection to confirm their identity.

\section{Sequence analysis}

DNASTAR was used for sequence analysis and DNAMAN was used to compare sequences. Information on sequences was obtained from GenBank (http://www.ncbi.nlm.nih.gov). The Homology of acquired sequences and known sequences were assessed using BLAST in order to identify mutations.

\section{Test of pathogenicity of MBC-resistance isolates on oilseed rape}

The pathogenicity of 74 isolates from different regions in Anhui was determined using the detached leaf inoculation test under greenhouse conditions (Liu et al., 2005). Wanyou 14 oilseed rape plants were grown in the greenhouse to the fourth true-leaf stage. Mycelia of S. sclerotiorum isolates were cultured on PDA for 3 days. Seven $\mathrm{mm}$ diameter agar discs were excised from the edges of growing colonies of each isolate and upended onto the detached leaves. Four replicate experiments were performed using eight leaves in each case. Treatment with blank agar discs was used as a control. All leaves were labeled and randomly arranged on wet gauze in containers that were collectively covered with transparent polyethylene bags. The leaves in the containers were incubated at $25^{\circ} \mathrm{C}$ in the dark. At $72 \mathrm{~h}$ after inoculation, the length and width of lesions were measured.

The isolates were separated into three types depending on the size of the disease spots on the leaves: strongly pathogenic, SPT, with an average diameter of disease spots $>3 \mathrm{~cm}$ after 72 h culture; weakly pathogenic, WPT, with an average diameter of disease spots $<1 \mathrm{~cm}$ after $72 \mathrm{~h}$ culture; and intermediate pathogenicity, IPT, with an average diameter of disease spots between 1 and $3 \mathrm{~cm}$ after $72 \mathrm{~h}$ culture (Huang and Li, 2009).

\section{RESULTS}

\section{Resistance of S. sclerotiorum from oilseed rape to MBC}

Analysis of the sensitivities to MBC showed that the 74 isolates of $\mathrm{S}$. sclerotiorum showed distinct differences in their $\mathrm{EC}_{50}$ values: 52 were sensitive to $\mathrm{MBC}$ with $\mathrm{EC}_{50}$ values $<0.5 \mathrm{mg} / \mathrm{L}$, 18 showed low resistance with $\mathrm{EC}_{50}$ values between 0.5 and $5 \mathrm{mg} / \mathrm{L}, 1$ (SX11) had moderate resistance with an $\mathrm{EC}_{50}$ value $=25.0854 \mathrm{mg} / \mathrm{L}$, and $3(\mathrm{SX} 2, \mathrm{SX} 4$ and NGA5) had high resistance 
with $\mathrm{EC}_{50}$ values of $214.9593,418.8048$ and $88.5202 \mathrm{mg} / \mathrm{L}$, respectively (Table 1). Resistant types accounted for $29.73 \%$ of the isolates: $24.32 \%$ were low resistance, $1.35 \%$ were moderate resistance, and $4.05 \%$ were high resistance types.

The results revealed a wide range of resistance to MBC in S. sclerotiorum isolates from the 13 growing areas with evidence of variation among regions. The SX population isolates from Shouxian showed the strongest resistance with $\mathrm{EC}_{50}$ values ranging from 0.0024 to 418.8048 $\mathrm{mg} / \mathrm{L}$, and a mean value of $73.4205 \mathrm{mg} / \mathrm{L}$. The NG population from Ningguo also showed strong resistance with $\mathrm{EC}_{50}$ values ranging from $0.0010 \mathrm{mg} / \mathrm{L}$ to $88.5202 \mathrm{mg} / \mathrm{L}$ and a mean of 4.6544 $\mathrm{mg} / \mathrm{L}$. The least resistance was shown by the NL population from Nanling with $\mathrm{EC}_{50}$ values ranging from $0.0112 \mathrm{mg} / \mathrm{L}$ to $0.0335 \mathrm{mg} / \mathrm{L}$ and a mean value of $0.0224 \mathrm{mg} / \mathrm{L}$ (Table 2).

Table 1. $\mathrm{EC}_{50}$ values for $\mathrm{MBC}$ against $\mathrm{S}$. sclerotiorum isolates from different regions of Anhui Province.

\begin{tabular}{|c|c|c|c|c|c|}
\hline Isolate & $\mathrm{EC}_{50}(\mathrm{mg} / \mathrm{L})$ & Isolate & $\mathrm{EC}_{50}(\mathrm{mg} / \mathrm{L})$ & Isolate & $\mathrm{EC}_{50}(\mathrm{mg} / \mathrm{L})$ \\
\hline LA1 & 0.7642 & JDB8 & 0.4445 & NGA4 & 0.2161 \\
\hline LAH & 0.5458 & JDA12 & 0.3004 & NGC8 & 0.0001 \\
\hline $\mathrm{NL}$ & 0.0335 & JDA11 & 0.3256 & NGA15 & 1.2818 \\
\hline NL2 & 0.0112 & JDA9 & 0.6816 & NGB5 & 2.8745 \\
\hline$X Z$ & 1.7340 & JDA16 & 0.0527 & NGA1 & 1.2915 \\
\hline $\mathrm{FC}$ & 0.0398 & JDA15 & 0.0009 & NGB-1-3 & 0.3810 \\
\hline HFD & 0.5558 & JDA7 & 0.0001 & NGA6 & 0.0060 \\
\hline $\mathrm{HF}$ & 0.4440 & JDA17 & 0.0935 & NGC6 & 0.0598 \\
\hline HFD-1-2 & 1.5575 & JDA13 & 0.9210 & NGA5 & 88.5202 \\
\hline HFD1 & 0.7627 & XJDB6 & 0.5099 & NGB1 & 0.0022 \\
\hline WH2 & 0.8595 & XJDA14 & 0.0001 & NGB-1-1 & 0.1886 \\
\hline WH & 0.0974 & XJDB8 & 0.5603 & NGA8 & 1.0890 \\
\hline WW2 & 0.3552 & XJDB7 & 0.0024 & NGB6 & 0.0769 \\
\hline WWB8 & 0.5449 & XJDB5 & 0.1957 & NGA9 & 0.0813 \\
\hline WW & 0.4882 & SX-9-1 & 0.3287 & NGC1 & 0.0010 \\
\hline WWD12 & 0.0232 & SX-A & 0.0501 & NGC10 & 0.0479 \\
\hline WJC-2 & 0.0863 & SX-3 & 1.0899 & NGB4 & 0.0153 \\
\hline TCD2 & 0.1417 & SX-5 & 0.4189 & NGB1-2 & 0.2970 \\
\hline TC-7 & 0.0124 & sX2 & 214.9593 & LJA-5-2 & 0.0361 \\
\hline TC-10 & 0.4281 & SX-11-1 & 0.0447 & LJD-5-1 & 0.1144 \\
\hline TCC1 & 0.0029 & sX6 & 0.0024 & LJD-5-3 & 0.3970 \\
\hline TC-a-1 & 0.3183 & SX4 & 418.8048 & LJE-3-1 & 0.0033 \\
\hline TC-E-3 & 0.7314 & SX11 & 25.0854 & & \\
\hline TC-E-1 & 0.1652 & NGC4 & 0.4911 & & \\
\hline TC-15 & 0.0013 & NGA7 & 0.8110 & & \\
\hline JDA10 & 0.0785 & NGC2 & 0.0105 & & \\
\hline
\end{tabular}

Table 2. The sensitivities of different populations of S. sclerotiorum to MBC.

\begin{tabular}{llcrr}
\hline Population & Origin & No.of isolates & EC $_{50}$ range dimension $(\mathrm{mg} / \mathrm{L})$ & Average \\
\hline JD & Jingde & 15 & $0.0001-0.9210$ & 0.2778 \\
NG & Ningguo & 21 & $0.0010-88.5202$ & 4.6544 \\
TC & Tongcheng & 8 & $0.0013-0.7314$ & 0.2252 \\
SX & Souxian & 9 & $0.0024-214.9593$ & 73.4205 \\
LJ & Lujiang & 4 & $0.0033-0.3907$ & 0.1377 \\
WW & Wuwei & 4 & $0.0232-0.5449$ & 0.3529 \\
HF & Hefei & 4 & $0.4440-0.7627$ & 0.8300 \\
NL & Nanling & 2 & $0.0112-0.0335$ & 0.0224 \\
WH & Wuhu & 2 & $0.0974-0.8595$ & 0.4785 \\
LA & Liuan & 2 & $0.5458-0.7642$ & 1.7340 \\
XZ & Xuanzhou & 1 & 0.0550 & 1.7340 \\
FC & Fanchang & 1 & 0.0398 & 0.0398 \\
WJ & Wangjiang & 1 & 0.0863 & 0.0863 \\
\hline
\end{tabular}

\section{Geographic distribution of MBC-resistant isolates of S. sclerotiorum}

We next examined the geographical distribution of the 22 resistant isolates across the 
13 regions of Anhui province. The proportion of resistant isolates varied among the different populations. Both Lu'an isolates were resistant while resistance in isolates from Hefei, Wuhu, and Shouxian was present in 75,50 and $44.44 \%$ of the samples, respectively. All isolates from Nanling, Xuanzhou, Fanchang, Wangjiang, and Lujiang were sensitive to MBC. The Ningguo isolates NGC8 and NGC1 were the most sensitive to $M B C$ with $E_{50}$ values of $0.0001 \mathrm{mg} / \mathrm{L}$ and 0.0010 $\mathrm{mg} / \mathrm{L}$, respectively. However, another isolate from this region, NGA5, had high resistance to MBC. Overall, the rate of resistance was $4.76 \%$ in Ningguo. Two highly resistant isolates were identified in the sample from Shouxian (SX2 and SX4), accounting for $22.22 \%$ of the isolates collected from this region. Our results indicate that isolates of $S$. sclerotiorum from many regions have developed resistance to $\mathrm{MBC}$, and that the level of resistance can vary within each area (Table 3).

Table 3. Survey of resistance in S. sclerotiorum populations and the proportion of isolates with resistance to MBC.

\begin{tabular}{llcccc}
\hline Isolate & Origin & No. of isolates & Resistant isolates & Sensitive isolate & Resistant proportion \\
\hline JD & Jingde & 15 & 4 & 11 & 26.67 \\
NG & Ningguo & 21 & 6 & 15 & 28.57 \\
TC & Tongcheng & 8 & 1 & 7 & 14.29 \\
LJ & Lujiang & 4 & 0 & 4 & 0 \\
SX & Shouxian & 9 & 4 & 5 & 44.44 \\
WW & Wuwei & 4 & 1 & 3 & 25.00 \\
HF & Hefei & 4 & 3 & 1 & 75.00 \\
WH & Wuhu & 2 & 1 & 2 & 50.00 \\
NL & Nanling & 2 & 0 & 1 & 0 \\
XZ & Xuanzhou & 1 & 0 & 1 & 0 \\
FC & Fanchang & 1 & 0 & 1 & 0 \\
WJ & Wangjiang & 1 & 0 & 0 & 0 \\
LA & Lu'an & 2 & 2 & 52 & 28.00 \\
Total & & 74 & 22 & & 0 \\
\hline
\end{tabular}

\section{$\beta$-tubulin gene sequences in MBC-resistant and MBC-sensitive isolates}

A $1.6 \mathrm{~kb}$ fragment of the $\beta$-tubulin gene was amplified by PCR from MBC-sensitive and MBCresistant isolates. Comparisons of the deduced amino acid sequences of the amplified products showed a point mutation in three high resistance isolates (NGA5, SX2, and SX4); in these isolates, the GAG at position 198 was replaced by GCG, resulting in an amino acid change from Glu to Ala. The $\beta$-tubulin gene in both the sensitive isolates and the low resistance isolates did not have the mutation (Figure 1). The results of our sequencing analysis indicated that mutation in the $\beta$-tubulin gene might be associated with MBC sensitivity in S. sclerotiorum isolates on oilseed rape in Anhui Province of Eastern China.

\section{Pathogenicity of MBC-resistant isolates of S. sclerotiorum}

Based on the results of our pathogenicity and MBC sensitivity tests, we examined MBC resistance and pathogenicity of the tested isolates on oilseed rape from different regions of Anhui (Table 4). We found that the three high resistance isolates, SX2 and SX4 were strongly pathogenic to oilseed rape (SPT), while NGA5 showed an intermediate level of pathogenicity (IPT). Furthermore, the moderate resistance SX11 isolate was a weakly pathogenic type (WPT). The MBC-sensitive isolates WW2, SX-5, and NGC6 varied in their pathogenicities and were SPT, IPT, and WPT, respectively. Similarly, the low resistance isolates LA1, HFD, and WH2 were WPT, IPT, and SPT, respectively. Thus, there was no correlation between MBC-resistance and pathogenicity in the $S$. sclerotiorum isolates tested (Table 4). The results suggest that the pathogenicity of $S$. sclerotiorum isolates on oilseed rape did not change with their MBC resistance. 


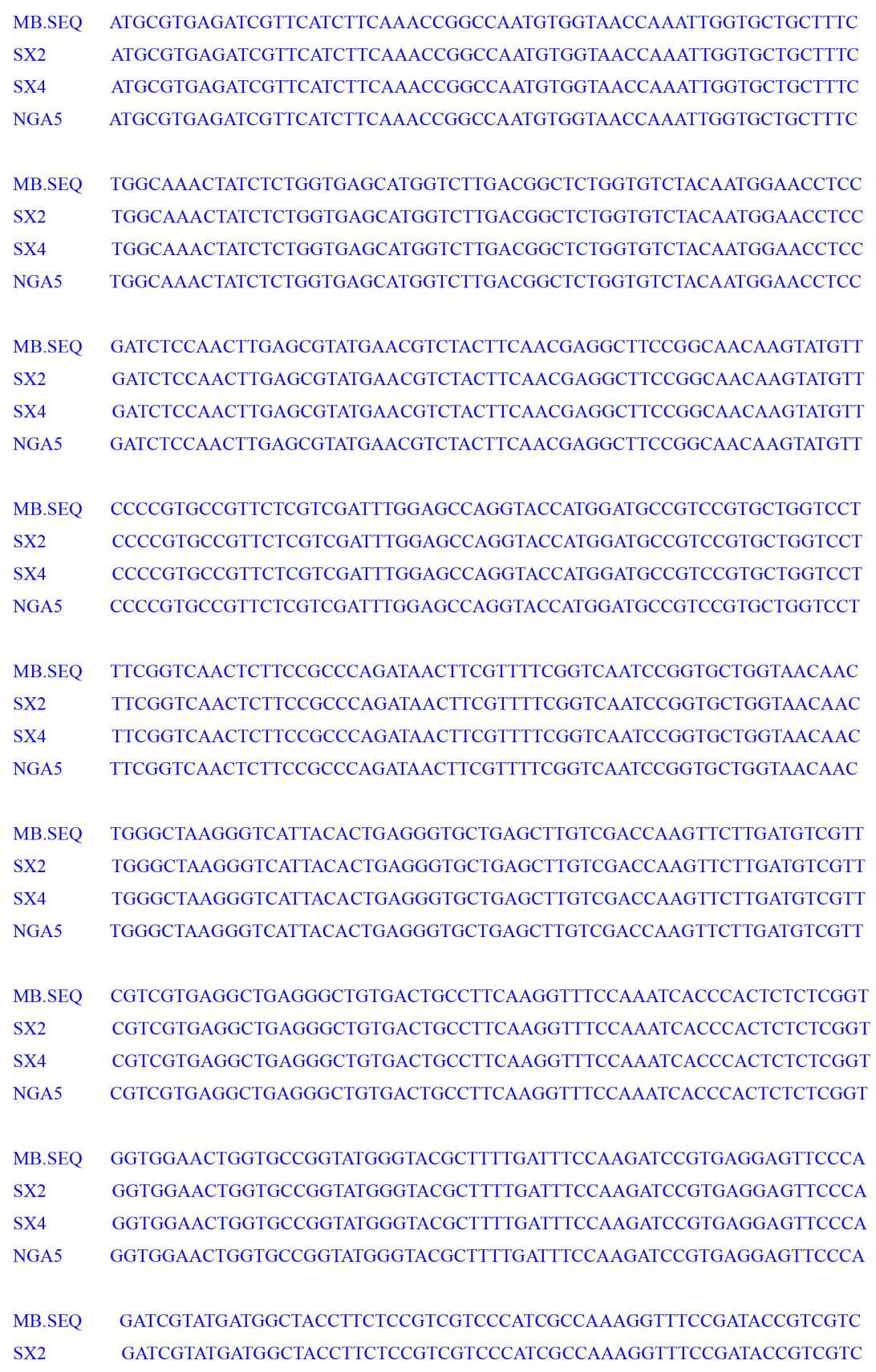

Figure 1. Nucleotide sequences of the $\beta$-tubulin gene from three isolates of Sclerotinia sclerotiorum from oilseed rape in Anhui Province of China. The MBC resistant isolates SX2, SX5, and NGA5 have a point mutation at position 198 in which GAG is replaced by GCG (boxed sequence). The deduced amino acid sequence from the MBC resistant isolates show they have an amino acid change from Glu to Ala as a result of this mutation.

Continued on next page 
Figure 1. Continued.

\begin{tabular}{|c|c|}
\hline SX4 & GATCGTATGATGGCTACCTTCTCCGTCGTCCCATCGCCAAAGGTTTCCGATACCGTCGTC \\
\hline NGA5 & GATCGTATGATGGCTACCTTCTCCGTCGTCCCATCGCCAAAGGTTTCCGATACCGTCGTC \\
\hline MB.SEQ & GAGCCATATAACGCTACTCTCTCTGTTCATCAATTGGTCGAGAACTCTGACGAGACCTTC \\
\hline SX2 & GAGCCATATAACGCTACTCTCTCTGTTCATCAATTGGTCGAGAACTCTGACGCGACCTTC \\
\hline SX4 & GAGCCATATAACGCTACTCTCTCTGTTCATCAATTGGTCGAGAACTCTGACGCGACCTTC \\
\hline NGA5 & GAGCCATATAACGCTACTCTCTCTGTTCATCAATTGGTCGAGAACTCTGACGCGACCTTC \\
\hline MB.SEQ & TGTATCGACAACGAGGCTCTCTACGACATTTGCATGAGAACCTTGAAGCTCAGCCACCCA \\
\hline SX2 & TGTATCGACAACGAGGCTCTCTACGACATTTGCATGAGAACCTTGAAGCTCAGCCACCCA \\
\hline SX4 & TGTATCGACAACGAGGCTCTCTACGACATTTGCATGAGAACCTTGAAGCTCAGCCACCCA \\
\hline NGA5 & TGTATCGACAACGAGGCTCTCTACGACATTTGCATGAGAACCTTGAAGCTCAGCCACCCA \\
\hline MB.SEQ & TCCTACGGAGATCTTAACCACTTGGTCTCCGCTGTCATGTCCGGTGTTACCACCTGTCTC \\
\hline SX2 & TCCTACGGAGATCTTAACCACTTGGTCTCCGCTGTCATGTCCGGTGTTACCACCTGTCTC \\
\hline SX4 4 & TCCTACGGAGATCTTAACCACTTGGTCTCCGCTGTCATGTCCGGTGTTACCACCTGTCTC \\
\hline NGA5 & TCCTACGGAGATCTTAACCACTTGGTCTCCGCTGTCATGTCCGGTGTTACCACCTGTCTC \\
\hline MB.SEQ & CGTTTCCCTGGTCAACTTAACTCAGATCTCCGAAAGTTGGCTGTCAACATGGTTCCATTC \\
\hline SX2 & CGTTTCCCTGGTCAACTTAACTCAGATCTCCGAAAGTTGGCTGTCAACATGGTTCCATTC \\
\hline SX4 & CGTTTCCCTGGTCAACTTAACTCAGATCTCCGAAAGTTGGCTGTCAACATGGTTCCATTC \\
\hline NGA5 & CGTTTCCCTGGTCAACTTAACTCAGATCTCCGAAAGTTGGCTGTCAACATGGTTCCATTC \\
\hline MB.SEQ & CCCCGTCTTCATTTCTTCATGGTTGGATTTGCTCCTTTGACCAGTCGTGGCGCACACTCT \\
\hline SX2 & CCCCGTCTTCATTTCTTCATGGTTGGATTTGCTCCTTTGACCAGTCGTGGCGCACACTCT \\
\hline SX4 & CCCCGTCTTCATTTCTTCATGGTTGGATTTGCTCCTTTGACCAGTCGTGGCGCACACTCT \\
\hline NGA5 & CCCCGTCTTCATTTCTTCATGGTTGGATTTGCTCCTTTGACCAGTCGTGGCGCACACTCT \\
\hline MB.SEQ & TTCCGTGCTGTTACTGTTCCAGAGTTGACCCAACAAATGTATGATCCTAAGAACATGATG \\
\hline SX2 & TTCCGTGCTGTTACTGTTCCAGAGTTGACCCAACAAATGTATGATCCTAAGAACATGATG \\
\hline SX4 & TTCCGTGCTGTTACTGTTCCAGAGTTGACCCAACAAATGTATGATCCTAAGAACATGATG \\
\hline NGA5 & TTCCGTGCTGTTACTGTTCCAGAGTTGACCCAACAAATGTATGATCCTAAGAACATGATG \\
\hline MB.SEQ & GCCGCTTCCGATTTCCGTAACGGTCGTTACTTAACCTGCTCTGCTATCTTCCGTGGTAAG \\
\hline SX2 & GCCGCTTCCGATTTCCGTAACGGTCGTTACTTAACCTGCTCTGCTATCTTCCGTGGTAAG \\
\hline SX4 & GCCGCTTCCGATTTCCGTAACGGTCGTTACTTAACCTGCTCTGCTATCTTCCGTGGTAAG \\
\hline NGA5 & GCCGCTTCCGATTTCCGTAACGGTCGTTACTTAACCTGCTCTGCTATCTTCCGTGGTAAG \\
\hline MB.SEQ & GTTTCCATGAAGGAGGTTGAGGACCAAATGCGCAATGTCCAAAACAAGAACTCTTCCTAC \\
\hline SX2 & GTTTCCATGAAGGAGGTTGAGGACCAAATGCGCAATGTCCAAAACAAGAACTCTTCCTAC \\
\hline SX4 & GTTTCCATGAAGGAGGTTGAGGACCAAATGCGCAATGTCCAAAACAAGAACTCTTCCTAC \\
\hline NGA5 & GTTTCCATGAAGGAGGTTGAGGACCAAATGCGCAATGTCCAAAACAAGAACTCTTCCTAC \\
\hline & CGAGTGGATCCCTAACAATGTCCAAACCGCCCTTTGCTCCATTCCTCCCCGTGGT \\
\hline
\end{tabular}


Figure 1. Continued.

\begin{tabular}{|c|c|}
\hline SX2 & TTCGTCGAGTGGATCCCTAACAATGTCCAAACCGCCCTTTGCTCCATTCCTCCCCGTGGT \\
\hline SX4 & TTCGTCGAGTGGATCCCTAACAATGTCCAAACCGCCCTTTGCTCCATTCCTCCCCGTGGT \\
\hline NGA5 & TTCGTCGAGTGGATCCCTAACAATGTCCAAACCGCCCTTTGCTCCATTCCTCCCCGTGGT \\
\hline MB.SEQ & CTCAAGATGTCCTCCACCTTCGTCGGTAACTCGACCTCCATCCAAGAACTCTTCAAGCGT \\
\hline SX2 & CTCAAGATGTCCTCCACCTTCGTCGGTAACTCGACCTCCATCCAAGAACTCTTCAAGCGT \\
\hline SX4 & CTCAAGATGTCCTCCACCTTCGTCGGTAACTCGACCTCCATCCAAGAACTCTTCAAGCGT \\
\hline NGA5 & CTCAAGATGTCCTCCACCTTCGTCGGTAACTCGACCTCCATCCAAGAACTCTTCAAGCGT \\
\hline MB.SEQ & GTCGGTGATCAATTCACTGCTATGTTCAGAAGAAAGGCTTTCTTGCATTGGTACACTGGT \\
\hline SX2 & GTCGGTGATCAATTCACTGCTATGTTCAGAAGAAAGGCTTTCTTGCATTGGTACACTGGT \\
\hline SX4 & GTCGGTGATCAATTCACTGCTATGTTCAGAAGAAAGGCTTTCTTGCATTGGTACACTGGT \\
\hline NGA5 & GTCGGTGATCAATTCACTGCTATGTTCAGAAGAAAGGCTTTCTTGCATTGGTACACTGGT \\
\hline MB.SEQ & AGGGTATGGACGAGATGGAGTTCACTGAAGCTGAGTCCAACATGAACGATTTGGTCTCC \\
\hline SX2 & AGGGTATGGACGAGATGGAGTTCACTGAAGCTGAGTCCAACATGAACGATTTGGTCTCC \\
\hline SX4 & AGGGTATGGACGAGATGGAGTTCACTGAAGCTGAGTCCAACATGAACGATTTGGTCTCC \\
\hline NGA5 & AGGGTATGGACGAGATGGAGTTCACTGAAGCTGAGTCCAACATGAACGATTTGGTCTCC \\
\hline MB.SEQ & GAGTACCAACAATACCAAGATGCCTCGATCTCTGAGGGAGAGGAGGAGTACGAAGAGGAA \\
\hline SX2 & GAGTACCAACAATACCAAGATGCCTCGATCTCTGAGGGAGAGGAGGAGTACGAAGAGGAA \\
\hline SX4 & GAGTACCAACAATACCAAGATGCCTCGATCTCTGAGGGAGAGGAGGAGTACGAAGAGGAA \\
\hline NGA5 & GAGTACCAACAATACCAAGATGCCTCGATCTCTGAGGGAGAGGAGGAGTACGAAGAGGAA \\
\hline MB.SEQ & GCCCCAATTGAGGGCGAGGAATA \\
\hline SX2 & GCCCCAATTGAGGGCGAGGAATA \\
\hline SX4 & GCCCCAATTGAGGGCGAGGAATA \\
\hline NGA5 & GCCCCAATTGAGGGCGAGGAATA \\
\hline
\end{tabular}

\section{DISCUSSION}

Oilseed rape is a major crop in China, and Anhui Province is one of the main production areas (Xu et al., 2014). Sclerotinia stem rot as a result of S. sclerotiorum infection is the principal disease of oilseed rape and has a significant impact on crop yields. In recent decades, MBC has been used widely for the control of this fungal disease; however, as resistance to this treatment has developed, it is important to assess the sensitivity of $S$. sclerotiorum to MBC in Anhui Province. Here, we examined 74 S. sclerotiorum isolates from 13 regions of Anhui Province and 
Table 4. Resistance type and pathogenicity category of isolates of S. sclerotiorum from different regions of Anhui province.

\begin{tabular}{|c|c|c|c|c|c|}
\hline Isolate & Resistance type ${ }^{a}$ & Pathogenicity category ${ }^{b}$ & Isolate & Resistance type ${ }^{a}$ & Pathogenicity category \\
\hline NL & S & SPT & NGB-1-3 & S & IPT \\
\hline NL2 & $\mathrm{S}$ & SPT & NGA6 & S & SPT \\
\hline$X Z$ & $\mathrm{~S}$ & SPT & NGC6 & $\mathrm{s}$ & WPT \\
\hline FC & $S$ & SPT & NGB1 & S & SPT \\
\hline $\mathrm{HF}$ & $\mathrm{s}$ & IPT & NGB-1-1 & $\mathrm{s}$ & IPT \\
\hline WH & $\mathrm{S}$ & SPT & NGB6 & S & SPT \\
\hline WW2 & $\mathrm{S}$ & IPT & NGA9 & $\mathrm{s}$ & IPT \\
\hline WW & $\mathrm{S}$ & SPT & NGC1 & S & SPT \\
\hline WWD12 & $S$ & IPT & NGC10 & S & IPT \\
\hline WJC-2 & $\mathrm{S}$ & SPT & NGB4 & S & IPT \\
\hline TCD2 & $\mathrm{S}$ & IPT & NGB-1-2 & $\mathrm{s}$ & IPT \\
\hline TC-7 & S & WPT & LJA-5-2 & S & IPT \\
\hline TC-10 & $\mathrm{s}$ & IPT & LJD-5-1 & s & SPT \\
\hline TCC1 & S & SPT & LJD-5-3 & $\mathrm{s}$ & IPT \\
\hline TC-a-1 & $S$ & IPT & LJE-3-1 & $S$ & SPT \\
\hline TC-E-1 & $\mathrm{S}$ & SPT & LA1 & LR & WPT \\
\hline TC-15 & $S$ & SPT & LAH & LR & IPT \\
\hline JDA10 & $S$ & SPT & HFD & LR & IPT \\
\hline JDB8 & S & SPT & HFD-1-2 & LR & SPT \\
\hline JDA12 & $\mathrm{S}$ & SPT & HFD-1 & LR & IPT \\
\hline JDA11 & S & SPT & WH2 & LR & SPT \\
\hline JDA16 & $S$ & SPT & WWB8 & LR & SPT \\
\hline JDA15 & S & SPT & TC-E-3 & LR & SPT \\
\hline JDA7 & $S$ & SPT & JDA9 & LR & SPT \\
\hline JDA17 & S & SPT & JDA13 & LR & SPT \\
\hline XJDA14 & $S$ & SPT & XJDB6 & LR & SPT \\
\hline XJDB7 & $\mathrm{s}$ & SPT & XJDB8 & LR & SPT \\
\hline XJDB5 & $\mathrm{S}$ & SPT & SX-3 & LR & SPT \\
\hline SX-9-1 & $S$ & SPT & NGA7 & LR & IPT \\
\hline SX-A & $S$ & SPT & NGA15 & LR & SPT \\
\hline SX-5 & $\mathrm{s}$ & IPT & NGB5 & LR & IPT \\
\hline SX-11-1 & $\mathrm{S}$ & SPT & NGA1 & LR & SPT \\
\hline SX6 & $\mathrm{s}$ & SPT & NGA8 & LR & SPT \\
\hline NGC4 & $S$ & IPT & SX11 & MR & WPT \\
\hline NGC2 & S & IPT & sX2 & HR & SPT \\
\hline NGA4 & $S$ & IPT & SX4 & $\mathrm{HR}$ & SPT \\
\hline NGC8 & $\mathrm{S}$ & SPT & NGA5 & $\mathrm{HR}$ & IPT \\
\hline
\end{tabular}

(a) Type of MBC-resistance: S, sensitive; LR, low resistance; MR, moderate resistance; HR, high resistance. (b) Pathogenicity types: WPT, weak; IPT, intermediate; SPT, strong.

found resistance to MBC in 22 isolates (29.7\%). To our knowledge, this is the first report of MBC resistance in S. sclerotiorum from Anhui Province of China. Although the current frequency of $\mathrm{MBC}^{\mathrm{MR}}$ and $\mathrm{MBC} \mathrm{HR}^{\mathrm{HR}}$ isolates was low $(5.41 \%)$, the rate of $\mathrm{MBC} \mathrm{LR}^{\mathrm{R}}$ isolates was quite high (24.32\%). Moreover, we also found that the pathogenicity of the isolates was not correlated with their MBCresistance, suggesting that the pathogenicity of $S$. sclerotiorum isolates on oilseed rape does not change with $\mathrm{MBC}$ resistance. In other words, the MBC resistant isolates of $\mathrm{S}$. sclerotiorum were as aggressive and fit as the original MBC sensitive isolates. In addition, we observed that the MBC resistant isolates showed comparable mycelial growth and sclerotia production as the sensitive isolates. This indicates that the MBC resistant isolates have strong reproductive fitness and have the capacity to develop into a dominant MBC resistant population in a field in a short time, which could lead to a failure in controlling Sclerotinia stem rot with MBC. Therefore, MBC resistance needs to be managed, and appropriate fungicide resistance management tactics need to be developed and employed, such as use of biological control agents, fungicide tank-mixing, or alternating MBC with other fungicides that have different modes of action; such tactics may aid the control of $S$. sclerotiorum on oilseed rape in Anhui. 
Our investigation also indicated that the frequency of resistant isolates varied among populations, i.e. the resistant proportion varied considerably among sampled regions. The difference may be connected with the known variability in the genetic structure of $S$. sclerotiorum populations in fields and regions (Kohn et al., 1991). Most S. sclerotiorum isolates in the field reproduce through sexual propagation, and have the potential of hybridization (Atallah et al., 2004). Another reason might be differences in the MBC application level in different regions. According to our survey, MBC application levels were high in regions or fields with a high ratio or level of resistant isolates, such as Hefei and Shouxian. The MBC-resistant strains were detected following the frequent use of MBC over a long period of time. In these areas, different strategies for MBC resistance management need to be adopted.

Our analysis demonstrated that highly resistant isolates had a point mutation at position 198 of their $\beta$-tubulin gene sequence that caused the substitution of glutamic acid with alanine. The $\beta$-tubulin gene in low resistance and sensitive isolates did not contain this mutation. This indicates that the mutation in the $\beta$-tubulin gene might be associated with high $\mathrm{MBC}$ resistance in S. sclerotiorum isolates. Although a similar mechanism has been reported in other fungi, such as Botrytis cinerea (Yarden and Katan, 1993), Venturia inaequelis (Koenraadt et al., 1992) , Aspergillus nidulans (Jung et al., 1992) and Neurospora crassa (Koenraadt and Jones, 1993), this is the first report of its occurrence in $S$. sclerotiorum isolates on oilseed rape. As the $\beta$-tubulin gene has been fully cloned and sequenced, we can quickly detect the presence of highly resistant groups and monitor their development in the field by a specific PCR assay. This advance therefore offers a significant opportunity to improve the control of Sclerotinia stem rot and the management of $\mathrm{MBC}$ resistance in Anhui Province.

\section{Conflicts of interest}

The authors declare no conflict of interest.

\section{ACKNOWLEDGMENTS}

Research is supported by the Commonwealth Specialized Research Fund of China Agriculture (Grant \#201103016) and the Oilseed Rape Industry System Fund of Anhui Province.

\section{REFERENCES}

Atallah ZK, Larget B, Chen X and Johnson DA (2004). High genetic diversity, phenotypic uniformity, and evidence of outcrossing in Sclerotinia Sclerotiorum in the Columbia Basin of Washington State. Phytopathology 94: 737-742.

Bardin SD and Huang HC (2001). Research on biology and control of Sclerotinia diseases in Canada. Can. J. Plant Pathol. 23: 88-98.

Boland GJ and Hall R (1994). Index of plant hosts of Sclerotinia sclerotiorum. Can. J. Plant Pathol. 16: 93-108.

Bolton MD, Thomma BP and Nelson BD (2006). Sclerotinia sclerotiorum (Lib.) de Bary: biology and molecular traits of a cosmopolitan pathogen. Mol. Plant Pathol. 7: 1-16.

Gao X, Han Q, Chen Y, Qin H, et al. (2014). Biological control of oilseed rape Sclerotinia stem rot by Bacillus subtilis strain Em7. Biocontrol Sci. Techn. 24: 39-52.

Huang J and Li GQ (2009). Double-stranded RNA elements and their association with pathogenicity of Sclerotinia sclerotiorum. Acta Phytopathol. Sin. 39: 30-35.

Jung MK, Wilder IB and Oakley BR (1992). Amino acid alterations in the ben A ( $\beta$-tubulin) gene of Aspergillus nidulans that confer benomyl resistance. Cell Motil. Cytoskeleton 22: 170-174.

Koenraadt $\mathrm{H}$ and Jones AL (1993). Resistance to benomyl conferred by mutation in codon 198 or 200 of the beta-tubulin gene 
of Neurospora crassa and sensitivity to diethofencarb conferred by codon 198. Phytopathology 83: 850-854.

Koenraadt H, Somerville SC and Jones AL (1992). Characterization of mutations in the beta-tubulin gene of benomyl-resistant fields strains of Venturia inaequalis and other plant pathogenic fungi. Phytopathology 82: 1348-1354.

Kohn LM, Stasovski E, Carbone I, Royerand J, et al. (1991). Mycelial incompatibility and molecular markers identify genetic variability in field populations of Sclerotinia sclerotiorum. Phytopathology 81: 480-485.

Letham DB, Huett DO and Trimboli DS (1976). Biology and control of Sclerotinia sclerotiorum in cauliflower and tomato crops in coastal New South Wales. Plant Dis. Rep. 60: 286-289.

Liu S, Wang H, Zhang J, Fitt BD, et al. (2005). In vitro mutation and selection of doubled-haploid Brassica napus lines with improved resistance to Sclerotinia sclerotiorum. Plant Cell Rep. 24: 133-44.

Lu G (2003). Engineering Sclerotinia sclerotiorum resistance in oilseed crops. Afr. J. Biotechnol. 2: 509-516.

Ma HX, Chen Y, Wang JX, Yu WY, et al. (2009). Activity of carbendazim, dimethachlon, iprodione, procymidone and boscalid against Sclerotinia stem rot in Jiangsu Province of China. Phytoparasitica 37: 421-429.

Perez F, Joliot A, Bloch-Gallego ZA, Zahraoui A, et al. (1992). Antennapedia homeobox as a signal for the cellular internalization and nuclear addressing of a small exogenous peptide. J. Cell Sci. 102: 717-722.

Purdy LH (1979). Sclerotinia sclerotiorum: history, disease, and symptomatology, host range, geographic distribution, and impact. Phytopathology 69: 875-880.

Shi ZQ, Zhou MG and Ye ZY (2000). Resistance of Sclerotinia sclerotiorum to carbendazim and dimethachlon. Chin. J. Oil Crop Sci. 22: 54-57.

Stewart CN and Via LE (1993). A rapid CTAB DNA isolation technique useful for RAPD finger printing and other PCR applications. Biotechniques 14: 748-750.

Wang Y, Hou YP, Chen CJ and Zhou MG (2014). Detection of resistance in Sclerotinia sclerotiorum to carbendazim and dimethachlon in Jiangsu Province of China. Australas. Plant Path. 43: 307-312.

Xu DF, Li XL, Pan YM, Dai YL, et al. (2014). Genetic diversity and pathogenicity differentiation of Sclerotinia sclerotiorum on rapeseed (Brassica napus L.) in Anhui Province, China. Genet. Mol. Res. 13: 10704-10713.

Yang JH, Pan YL, Zhu GM and Zhou YM (2004). Mechanism of resistance of Sclerotinia sclerotiorum to carbendazim and diethofencarb. Acta Phytophyl. Sin. 31: 74-78.

Yarden $\mathrm{O}$ and Katan T (1993). Mutations leading to substitutions at amino acids 198 and 200 of beta-tubulin that correlate with benomyl-resistance phenotypes of field strains of Botrytis cinerea. Phytopathology 83: 1478-1483.

Zhang HJ, Wu Q, Cao S, Zhao TY, et al. (2014). A novel protein elicitor (SsCut) from Sclerotinia sclerotiorum induces multiple defense responses in plants. Plant Mol. Biol. 86: 495-511

Zhang XL, Sun XM and Zhang GF (2003). Preliminary report on the monitoring of the resistance of Sclerotinia libertinia to carbendazim and its internal management. Chin. J. Pest. Sci. Admin. 24: 18-22.

Zhao J and Meng J (2003). Detection of loci controlling seed glucosinolate content and their association with Sclerotinia resistance in Brassica napus. Plant Breed. 122: 19-23. 